\title{
Rabaska
}

Revue d'ethnologie de l'Amérique française

[COMMISSION DES BIENS CULTURELS]. Les Chemins de la mémoire. Monuments et sites historiques du Québec. Québec, Les Publications du Québec, Tome I, 1990, 540 p. ISBN 2-551-14145-1. Tome II, 1991, 565 p. ISBN 2-551-14570-8. Monuments et sites historiques du Québec. Supplément 1987-1999. Québec, Commission des biens culturels, 2001, 34 p. Biens mobiliers du Québec. Québec, Les Publications du Québec, Tome III, 1999, 428 p. ISBN 2-551-18161-5

\section{Georges Gauthier-Larouche}

\section{Numéro 1, 2003}

URI : https://id.erudit.org/iderudit/201616ar

DOI : https://doi.org/10.7202/201616ar

Aller au sommaire du numéro

Éditeur(s)

Société québécoise d'ethnologie

ISSN

1703-7433 (imprimé)

1916-7350 (numérique)

Découvrir la revue

Citer ce compte rendu

Gauthier-Larouche, G. (2003). Compte rendu de [[COMMISSION DES BIENS CULTURELS]. Les Chemins de la mémoire. Monuments et sites historiques du Québec. Québec, Les Publications du Québec, Tome I, 1990, 540 p. ISBN 2-551-14145-1. Tome II, 1991, 565 p. ISBN 2-551-14570-8. Monuments et sites historiques du Québec. Supplément 1987-1999. Québec, Commission des biens culturels, 2001, 34 p. Biens mobiliers du Québec. Québec, Les Publications du Québec, Tome III, 1999, 428 p. ISBN 2-551-18161-5]. Rabaska, (1), 165-168. https://doi.org/10.7202/201616ar d'utilisation que vous pouvez consulter en ligne. 
[Commission des Biens Culturels]. Les Chemins de la mémoire. Monuments et sites historiques du Québec. Québec, Les Publications du Québec, Tome I, 1990, 540 p. ISBN 2-551-14145-1. Tome II, 1991, 565 p. ISBN 2-551-14570-8. Monuments et sites historiques du Québec. Supplément 1987-1999. Québec, Commission des biens culturels, 2001, 34 p. Biens mobiliers du Québec. Québec, Les Publications du Québec, Tome III, 1999, 428 p. ISBN 2-551-18161-5.

En prenant connaissance de cette œuvre, l'on ne peut s'empêcher d'évaluer le chemin parcouru depuis la création de la Commission des monuments historiques en 1922 et la Commission des biens culturels qui lui succéda un demi-siècle plus tard.

Quelques années après sa fondation, la Commission des monuments historiques publiait Les Vieilles Églises de la province de Québec (1925) et Vieux Manoirs, vieilles maisons (1927), deux livres assez remarquables pour l'époque, compte tenu du fait que la compilation et la rédaction des textes étaient redevables, semble-t-il, à un seul chercheur, en l'occurrence Pierre-Georges Roy, premier secrétaire de la Commission des monuments historiques.

Le but poursuivi par celle-ci était "d'inspirer le respect de la vieille maison, l'amour de l'humble demeure, le culte du rustique foyer », d'inculquer aussi à la population, le rejet du «bungalow insignifiant » (ce mot emprunté à l'anglais bungelow prend ses racines dans bangalo et d'abord dans bangla, « maison du Bengale ». Le peuple non aryen Bang fut appliqué à une région de l'Inde nommée Bengale ; Alain Rey, Dictionnaire historique de la langue française, 1998) et, considération importante probablement oubliée, « ne pas copier l'ancien, mais garder ce qui reste de l'héritage de nos ancêtre»; somme toute, nos devanciers voyaient juste en voulant développer l'esprit plutôt que la lettre. Ainsi, dès le début, l'orientation générale à ce genre d'ouvrages était 
pratiquement arrêtée, et aujourd'hui, ces deux publications des années 1920 peuvent être considérées comme formant un tout, malgré leur édition séparée.

Or ce genre d'ouvrage, nous le retrouvons, quelque soixante-cinq années plus tard, dans les tomes 1 et 2 de l'œuvre intitulée Les Chemins de la mémoire, publiée en 1990 et 1991 par la Commission des biens culturels du Québec et dans le supplément de trente-quatre pages (2001), lesquels ont comme soustitre: Monuments et sites historiques du Québec. La différence essentielle entre les deux époques tient dans les dimensions considérablement élargies du projet de la Commission des biens culturels et dans le but qu'elle poursuit, savoir : conférer, selon les termes de la Loi sur les biens culturels (1972), un statut légal de sauvegarde, de protection et d'entretien, aux monuments répertoriés, de même qu'aux sites, emplacements ou domaines méritant la même considération. Incidemment, sur les cinq cents biens immobiliers répertoriés dans les deux premiers tomes, les sites entrent dans une proportion de $9 \%$; il s'agit de quelques arrondissements historiques, mais surtout de sites archéologiques et institutionnels, outre quelques sites de pêche. Les monuments, ce sont des constructions civiles, conventuelles, domestiques, militaires et industrielles dont l'éventail est très large. On peut noter le grand format de chaque volume, le nombre de collaborateurs dépassant quatrevingts dans les deux premiers tomes, environ quinze cents photos, dont certaines en couleur, des descriptions étoffées, tout cela témoignant, il va sans dire, de l'ampleur de l'entreprise, comparée à celle de 1925 .

Le tome 1 concerne les régions de Mauricie-Bois-Francs, de Québec et Chaudière-Appalaches et de l'Est du Québec à partir du Saguenay. Chaque région, décrite par un texte élaboré, est divisée en sous-régions que les éditeurs du projet ont appelé « circuits »; ils sont au nombre de dix, et chacun de ces circuits est précédé également par un texte compréhensif et par un plan de localisation des lieux concernés. La table des matières, placée au début, est complétée, à la fin de l'ouvrage, par une liste des monuments et des sites, ainsi que par une liste des lieux, énumérés dans les deux cas, par ordre alphabétique.

Le tome 2, en tous points conçu comme le premier, est rédigé, sauf quelques noms, par une équipe différente de celle de Québec et couvre le territoire situé à l'ouest de Trois-Rivières. L'île de Montréal accapare la plus grande part des études; viennent ensuite les régions de l'Estrie, de la Montérégie, de Lanaudière, de Laval-Laurentides, de l'Outaouais et de l'Abitibi-Témiscamingue. Comme dans le premier tome, les titres des ouvrages qui ont servi de sources pour la rédaction des textes sont distribués à la fin des rubriques.

Malgré la présentation matérielle impeccable dans les deux tomes et l'excellence des textes, on peut déplorer l'absence d'une bibliographie qui 
aurait réuni en quelques pages les titres des ouvrages consultés. Une autre lacune à relever serait de ne pas avoir énuméré les articles de chaque collaborateur. Si on l'eût fait, tout lecteur aurait aperçu, au premier coup d'œil, l'apport respectif des spécialistes. Malheureusement, nous n'avons droit qu'à une liste sèche des auteurs, placée sur un des revers de la jaquette des livres; et si l'on veut connaître la contribution de chacun, il faut faire l'exercice soi-même. Quoi qu'il en soit, il demeure impossible de tenir compte des textes de chacun pour tenter de déceler des erreurs de fait ou des lacunes, sans doute rares, ici et là ; chaque auteur, qu'il soit historien, historien de l'art ou de l'architecture, ethnologue, architecte ou appartenant à quelque autre spécialité, aborde son étude propre, selon sa culture personnelle. Néanmoins, en dépit de la variété des points de vue offerts par la pratique d'une discipline, la composition, dans l'ensemble des deux tomes, est relativement uniforme, ce qui lui confère une unité certaine, trop souvent absente, d'ailleurs, dans ces sortes d'ouvrages collectifs.

Le troisième tome, sous-titré Biens mobiliers du Québec, bien qu'un peu moins abondant que les deux premiers tomes, n'en est pas moins dense. Notons que les responsables de la publication ont corrigé les lacunes que nous avons relevées dans les deux premiers tomes. En effet, l'apport respectif de la quarantaine d'auteurs est fourni sur une seule page ; de même, la liste des ouvrages et des articles cités est donnée, non pas à la fin du volume comme on pourrait s' $y$ attendre, mais après la première partie qui est la plus importante de l'ouvrage; et, à la fin de chaque article, les références sont réduites à leur plus simple expression, mais suffisantes pour repérer le titre en entier dans la bibliographie. Un index onomastique et la localisation des biens terminent le livre.

La catégorie la plus abondante concerne les œuvres et objets d'art, pour la très grande majorité religieux, associés au culte catholique; mais elle comprend aussi notamment trois orgues, le char de l'Agriculture et la statue équestre de Saint-Georges érigée devant l'église beauceronne de SaintGeorges, toutes deux, œuvres de Louis Jobin, la statue Notre-Dame du Saguenay, du même sculpteur - dont le texte explicatif, paru en 1990 dans le premier tome, est revu et augmenté ici —, le décor intérieur de la maison Porteous à l'Île d'Orléans, une collection de dessins de Théophile Hamel, la maison et les meubles de l'architecte montréalais Ernest Cormier, un portrait au pastel de Wilfrid Laurier par Suzor-Côté, les peintures de la chapelle SaintAntoine-de-Padoue de Lac-Bouchette par Charles Huot, et aussi divers décors intérieurs de chapelles. Comme les œuvres religieuses ne peuvent pas toutes être illustrées, celles non choisies sont néanmoins énumérées à la fin des articles, le cas échéant. 
Après les œuvres d'art, suivent les fonds et les documents d'archives d'institutions universitaires, tels ceux du Séminaire de Rimouski et de l'université Bishop, de même que ceux d'hommes politiques, d'écrivains célèbres, de chercheurs, d'architectes, et même d'entrepreneurs industriels; bref, une catégorie riche et très intéressante à découvrir : notons, dans ce groupe, le fonds d'archives de l'architecte Ernest Cormier, la collection Robert-LionelSéguin et même les livres de compte du Séminaire de Québec (1674-1934).

La troisième et dernière catégorie, apparemment la moins abondante du volume, représente à elle seule 4700 inscriptions au Registre des biens culturels du Québec; outils de la forge Asselin, et outils et meubles de la chalouperie Godbout, toutes deux à l'île d'Orléans; outils de la menuiserie Cauchon à Rivière-Malbaie ; outillage de la fromagerie Perron de Saint-Prime et les biens meubles de la maison Stuart-Henry à Québec.

On aurait pu établir une catégorie spéciale pour les portails du Kensington Apartments, le violon Stradivarius Des Rosiers et un archet François Tourte utilisés par le regretté Arthur Leblanc, et maintenant par la violoniste Angèle Dubeau, la collection de poissons fossiles de Miguasha, en Gaspésie, et le drapeau fleurdelysé québécois adopté le 21 janvier 1948.

Les auteurs du troisième tome ont livré un ouvrage dense, de même excellente qualité que celle des deux premiers tomes.

Somme toute, cet inventaire d'une partie de nos biens culturels titré Les Chemins de la mémoire est un apport d'envergure à la connaissance de la culture matérielle du Québec, lequel servira de façon permanente à toute personne intéressée aux legs du passé que le temps n'aura pas démolis.

Georges Gauthier-Larouche

Québec 